\title{
ON THE EVALUATION OF SPECTRAL PROJECTIONS ONTO ABSOLUTELY CONTINUOUS PARTS OF A CONTRACTION
}

\author{
ALEXEY V. RYBKIN
}

(Communicated by Paul S. Muhly)

\begin{abstract}
In terms of the characteristic function of the contraction the norms of the spectral projections onto its absolutely continuous component are evaluated. The row of examples is considered. The exposition is conducted in the framework of the Sz.-Nagy-Foias model.
\end{abstract}

Let $T$ be a completely nonunitary (for terms used without explanation, see [6]) contraction in the Hilbert space $\mathfrak{H}$ and let $\mathfrak{U} \equiv \operatorname{clos}\left(I-T^{*} T\right)^{1 / 2}$ be the defect subspace of $T$; let $S(z)$ be the characteristic function (c.f.) of the contraction $T$ and $U$ be its minimal unitary dilation in the Hilbert space $\mathfrak{R}(\mathfrak{R} \supset \mathfrak{H})$. As the model space $\mathfrak{R}$ we take $\mathfrak{R} \stackrel{\text { def }}{=} L_{2}\left({ }_{S}^{I} S_{I}^{*}\right)$ (so-called Pavlov transcription $[3,4])$, the Hilbert space of two-component function $f(\xi)=\left(f_{0}(\xi), f_{1}(\xi)\right)$ on the unit circle $\mathbf{T}, \xi=e^{i \varphi}$, taking values in $\mathfrak{U} \oplus \mathfrak{U}$ with norm (1)

$$
\langle\langle f\rangle\rangle^{2}=\int_{-\pi}^{\pi}\left\langle\left(\begin{array}{cc}
I & S^{*}(\xi) \\
S(\xi) & I
\end{array}\right)\left(\begin{array}{l}
f_{0}(\xi) \\
f_{1}(\xi)
\end{array}\right),\left(\begin{array}{l}
f_{0}(\xi) \\
f_{1}(\xi)
\end{array}\right)\right\rangle_{\mathfrak{U} \oplus \mathcal{U}} d m, \quad d m=\frac{d \varphi}{2 \pi} .
$$

It is assumed that the set of two-component functions has been factored by the set of elements with norm equal to zero. In this case the original space $\mathfrak{H}$ is identified as the set of all vectors $\left(f_{0}, f_{1}\right)$ such that $f_{+} \equiv S f_{0}+f_{1} \in H_{+}^{2}(\mathfrak{U})$, $f_{-} \equiv f_{0}+S^{*} f_{1} \in H_{-}^{2}(\mathfrak{U})$, where $H_{ \pm}^{2}(\mathfrak{U})$ are the vector-valued Hardy classes into the interior $(+)$ (the exterior $(-))$ of the disk.

2

We restrict at first our considerations on the contractions $T$ the spectrum of which $\sigma(T)$ is absolutely continuous $\left(\sigma(T)=\sigma_{\mathrm{ac}}(T)\right)$ and $\sigma_{\mathrm{ac}}(T) \subseteq \mathbf{T}$. It

Received by the editors October 23, 1989 and, in revised form, March 26, 1990.

1980 Mathematics Subject Classification (1985 Revision). Primary 47A20, 47A45, 47A30; Secondary 30D40.

Key words and phrases. Contraction, characteristic operator-valued function, functional model spectral projection, boundary behaviour. 
means (see $[4,5])$ that the c.f. of $T$ is a purely outer, i.e., $\operatorname{clos} S H_{+}^{2}(\mathfrak{U})=H_{+}^{2}(\mathfrak{U})$, and the linear manifold

$$
\widetilde{\mathfrak{H}} \equiv\left\{f: f=P_{\mathfrak{H}}\left(\begin{array}{c}
h \\
-S h
\end{array}\right), h \in L_{2}(\Delta)\right\}, \quad \Delta \equiv I-S^{*} S,
$$

is dense in $\mathfrak{H}$ (here $P_{\mathfrak{H}}$ is the orthogonal projection in $\mathfrak{R}$ onto $\mathfrak{H}$ ).

The following vector-valued variant of the Carleson imbedding theorem will be used (see [2]).

Proposition. Let $\mu$ be a Carleson measure in the unit disk $\mathbf{D}$, i.e.,

$$
C^{2} \equiv \operatorname{Sup}\left\{\int_{|z| \leq|\xi|<1} \frac{1-|z|^{2}}{\left|1-\bar{\xi}_{z}\right|^{2}} d \mu(\xi): z \in \operatorname{Supp} \mu\right\}<\infty .
$$

Then for each $f \in H_{+}^{2}(\mathfrak{U})$, where $\mathfrak{U}$ is a Hilbert space,

$$
\|f\|_{L_{2}(d \mu, \mathfrak{U})} \leq 4 \sqrt{2} c\|f\|_{H_{+}^{2}(\mathfrak{U})} .
$$

A Jordan contour is clos $\mathbf{D}$ is called Carleson contour if its arclength measure satisfies condition (2).

We will say that a point $\xi \in \mathbf{T}$ is a conditionally regular point of $T$ if there exists a Carleson curve $\Gamma_{\xi}$ in clos D with one of the ends in $\xi$ such that $\operatorname{Sup}\left\{\left\|S^{-1}(z)\right\|: z \in \Gamma_{\xi}\right\}<\infty$. The complement of conditionally regular points will be called the set of essential spectral singularities $\sigma_{\text {ess }}$ of $T$.

It immediately follows from the definition that $\sigma_{\text {ess }} \subseteq \sigma_{\text {ss }}$, where $\sigma_{\text {ss }}$ is a set of spectral singularities $[1,4]$, i.e., points for which

$$
\varlimsup \varlimsup^{-1}(z) \|=\infty, \quad z \rightarrow \xi, \quad z \in \mathbf{D} .
$$

Theorem. Let $T$ be a contraction and $\sigma(T)=\sigma_{\mathrm{ac}}(T) \subseteq \mathbf{T}$. Then the spectral projection $\mathfrak{P}_{\omega}$ corresponding to the arc $\omega=\left[\xi_{1}, \xi_{2}\right] \subset \sigma(T), \xi_{1} \neq \xi_{2}$, is bounded if $\xi_{1}, \xi_{2} \notin \sigma_{\text {ess }}$. In this case

$$
\left\|\mathfrak{P}_{\omega}\right\| \leq 1+\frac{16}{\pi} \inf _{\Gamma_{\omega}}\left\{C^{2}\left(\Gamma_{\omega}\right) \operatorname{Sup}_{z \in \Gamma_{\omega}}\left\|S^{-1}(z)\right\|\right\},
$$

where $\Gamma_{\omega}$ is any Carleson contour with endpoints $\xi_{1}, \xi_{2}$.

Proof. On the dense set $\widetilde{\mathfrak{H}}$ the spectral projection is obeying the following rule [5]:

$$
\mathfrak{P}_{\omega} f=\mathfrak{P}_{\omega} P_{\mathfrak{H}}\left(\begin{array}{c}
h \\
-S h
\end{array}\right)=P_{\mathfrak{H}}\left(\begin{array}{c}
\chi_{\omega} h \\
-S \chi_{\omega} h
\end{array}\right)
$$


where $\chi_{\omega}$ is the characteristic function of $\omega$. If $f=\left(f_{0}, f_{1}\right) \in \widetilde{\mathfrak{H}}$ and $g=$ $\left(g_{0}, g_{1}\right) \in \mathfrak{H}$ :

$$
\begin{aligned}
\left\langle\left\langle\mathfrak{P}_{\omega} f, g\right\rangle\right\rangle & =\int_{-\pi}^{\pi}\left\langle\left(\begin{array}{cc}
I & S^{*} \\
S & I
\end{array}\right) P_{\mathfrak{H}}\left(\begin{array}{c}
\chi_{\omega} h \\
-S \chi_{\omega} h
\end{array}\right),\left(\begin{array}{l}
g_{0} \\
g_{1}
\end{array}\right)\right\rangle d m \\
& =\int_{-\pi}^{\pi}\left\langle\left(\begin{array}{c}
\chi_{\omega} h-P_{H_{+}^{2}} \chi_{\omega} \Delta h \\
-S \chi_{\omega} h
\end{array}\right),\left(\begin{array}{l}
g_{-} \\
g_{+}
\end{array}\right)\right\rangle d m \\
& =\int_{-\pi}^{\pi} \chi_{\omega}\left(\left\langle h, g_{-}\right\rangle+\left\langle S h, g_{+}\right\rangle\right) d m \\
& =\int_{\omega}\left\langle\Delta h, g_{0}\right\rangle d m .
\end{aligned}
$$

It is easy to check by direct calculation that

$$
\left\langle\Delta h, g_{0}\right\rangle=\left\langle f_{+}, g_{+}\right\rangle+\left\langle\Delta f_{0}, g_{0}\right\rangle-\left\langle S^{-1} f_{+}, g_{-}\right\rangle \text {. }
$$

Using this relation, we have:

$$
\left\langle\left\langle\mathfrak{P}_{\omega} f, g\right\rangle\right\rangle=\int_{\omega}\left(\left\langle f_{+}, g_{+}\right\rangle+\left\langle\Delta f_{0}, g_{0}\right\rangle\right) d m-\int_{\omega}\left\langle S^{-1} f_{+}, g_{-}\right\rangle d m .
$$

The first integral in the right-hand side of (4) is the bilinear form of spectral projection of dilation $U$ and therefore

$$
\begin{aligned}
\mid \int_{\omega} & \left(\left\langle f_{+}, g_{+}\right\rangle+\left\langle\Delta f_{0}, g_{0}\right\rangle\right) d m \mid \\
& =\left|\int_{\omega}\left(\left\langle f_{-}, g_{0}\right\rangle+\left\langle f_{+}, g_{1}\right\rangle\right) d m\right| \\
& =\left|\int_{-\pi}^{\pi}\left\langle\left(\begin{array}{cc}
I & S^{*} \\
S & I
\end{array}\right) \chi_{\omega} f, g\right\rangle d m\right| \\
& \leq\langle\langle f\rangle\rangle \cdot\langle\langle g\rangle\rangle .
\end{aligned}
$$

To estimate the second integral in (4) we deform the arc $\omega$ into some Carleson contour $\Gamma_{\omega}$ on which $\left\|S^{-1}(z)\right\|$ is bounded:

$$
\begin{aligned}
\left|\int_{\omega}\left\langle S^{-1} f_{+}, g_{-}\right\rangle d m\right| \\
\quad=\left|\frac{1}{2 \pi i} \int_{\Gamma_{\omega}}\left\langle S^{-1} f_{+}, g_{-}\right\rangle \frac{d z}{z}\right| \\
\quad \leq \frac{1}{2 \pi}\left(\int_{\Gamma_{\omega}}\left\|S^{-1} f_{+}\right\|_{\mathfrak{U}}^{2}|d z|\right)^{1 / 2} \cdot\left(\int_{\Gamma_{\omega}}\left\|z \overline{g_{-}\left(z^{*}\right)}\right\|_{\mathfrak{U}}^{2}|d z|\right)^{1 / 2} .
\end{aligned}
$$

By applying the Carleson theorem and the simple inequalities $\left\|f_{ \pm}\right\|_{H_{ \pm}^{2}(\mathfrak{U})} \leq\langle\langle f\rangle\rangle$ we have

$$
\left|\int_{\omega}\left\langle S^{-1} f_{+}, g_{-}\right\rangle d m\right| \leq \frac{16}{\pi} C^{2}\left(\Gamma_{\omega}\right) \operatorname{Sup}_{z \in \Gamma_{\omega}}\left\|S^{-1}(z)\right\| \cdot\langle\langle f\rangle\rangle\langle\langle g\rangle\rangle .
$$


From (5), (6) and the density of $\widetilde{\mathfrak{H}}$ in $\mathfrak{H}$, we arrive at the required result. Q.E.D.

Remark 1. The result and proof of the theorem, obviously, are preserved if $\omega=\bigcup_{j} \omega_{j}$, where $\left\{\omega_{j}\right\}$ are arcs (in this case $\Gamma_{\omega}=\bigcup_{j} \Gamma_{\omega_{j}}$ and generally speaking $\Gamma_{\omega}$ is nonconnected). The cases $\omega=\sigma(T), \omega=\{\xi\}$ are trivial: $\mathfrak{P}(\sigma)=I, \mathfrak{P}(\{\xi\})=0$.

Remark 2. In the general case when c.f. $S$ is not purely outer one must test the boundedness of $\mathfrak{P}_{\omega}$ in two steps. One first considers the boundedness conditions for the projection of $\mathfrak{H}_{\text {in }}+\mathfrak{H}_{\text {out }}(\omega)$ onto $\mathfrak{H}_{\text {out }}(\omega)$, where $\mathfrak{H}_{\text {in }}\left(\mathfrak{H}_{\text {out }}\right)$ is the maximal hyperinvariant subspace of $T$ where the pure part of the c.f. of the restriction $T \mid \mathfrak{H}_{\text {in }}\left(T \mid \mathfrak{H}_{\text {out }}\right)$ is an inner (outer) function [4,6]; $\mathfrak{H}_{\text {out }}(\omega)=$ clos Rang $\mathfrak{P} \omega^{\mathfrak{H}}$. At this step we may use well-known separation conditions for $\mathfrak{H}_{\text {in }}, \mathfrak{H}_{\text {out }}($ see $[4,5])$. After this one applies our theorem to the projection of $\mathfrak{H}_{\text {out }}$ onto $\mathfrak{H}_{\text {out }}(\omega)$.

3

We will illustrate our theorem by some examples, which show that $\sigma_{\text {ess }}$ is significantly poorer than $\sigma_{\mathrm{ss}}$.

Example 1. Let $T$ be a contraction with $\operatorname{dim} \mathfrak{U}=1$ (so-called scalar case). Let $T$ have the following outer c.f.

$$
S(z)=e^{-u(z)}, \quad \operatorname{Re} u(z)=\int_{-\pi}^{\pi} P_{z}(t) f(t) d t, \quad P_{z}(t) \equiv \frac{1}{2 \pi} \frac{1-|z|^{2}}{\left|e^{i t}-z\right|^{2}},
$$

where

$$
f(t)= \begin{cases}-t, & -\pi \leq t \leq 0 \\ t^{-1 / 2}, & 0<t \leq \pi\end{cases}
$$

Obviously, $\sigma(T)=\sigma_{\mathrm{ac}}(T)=\mathbf{T}$. By direct calculation it is easy to show that $\varlimsup\left|S^{-1}(z)\right|=\infty$, i.e., $1 \in \sigma_{\text {ss }}$. But along the tangential path $z=r e^{i \varphi}, \varphi<0$, $|\varphi|^{-3 / 2}(1-r) \rightarrow 0$, which, obviously, is a Carleson curve, we have

$$
\lim \left|S^{-1}(z)\right|=1, \quad z \rightarrow 1 .
$$

Therefore $1 \notin \sigma_{\text {ess }}$ (but $1 \in \sigma_{\text {ss }}$ ). Hence, we constructed the contraction $T$ such that $\sigma_{\text {ess }}=\varnothing$ and, therefore, $\mathfrak{P}_{\omega}$ is bounded for each arc $\omega$. It is necessary to note, however, that $T$ is not a spectral operator in the sense of Dunford, because $\sigma_{\text {ss }}=\{1\} \neq \varnothing$, and therefore $\operatorname{Sup}\left\{\left\|\mathfrak{P}_{\omega}\right\|: \omega \subset \mathbf{T}\right\}=\infty$.

Example 2. Let us consider contraction $T$ with $\operatorname{dim} \mathfrak{U}=1$ and c.f.

$$
S(z)=\prod_{k} \bar{z}_{k} \frac{\xi_{k}-z}{1-\bar{z}_{k} z}, \quad \xi_{k}=\frac{z_{k}}{\left|z_{k}\right|},
$$

where $\left\{z_{k}\right\}$ is Frostman sequence in $\mathbf{D}$ (i.e., $\operatorname{Sup}\left\{\sum_{k} 1-\left|z_{k}\right| /\left|1-\xi z_{k}\right|: \xi \in \mathbf{T}\right\}$ $<\infty)$. It is easy to check that $S(z)$ is an outer function. Obviously $\left\{\xi_{k}\right\} \subseteq \sigma_{\text {ess }}$ 
and a cluster set for $\left\{\xi_{k}\right\}$ belongs to $\sigma_{\text {ss }}$. But it is easy to see that the Frostman condition guarantees that the function $\left|S^{-1}(z)\right|$ is bounded along radii at the points of the cluster set. Hence $\sigma_{\text {ss }}=\operatorname{clos}\left\{\xi_{k}\right\}$, but $\sigma_{\text {ess }}=\left\{\xi_{k}\right\}$.

The bilateral estimation for projection in the scalar case was obtained by V. I. Vasyunin (see [1]) in terms of solution of the Carona problem. The following example shows that the conditions of the theorem in the general case are not necessary for the boundedness of $\mathfrak{P}_{\omega}$.

Example 3. Let $T=T_{1} \oplus T_{2}$, where $T_{i}$ is a contraction in $\mathfrak{H}_{i}$ and $\operatorname{dim} \mathfrak{U}_{i}=1$ $(i=1,2)$. The c.f. $S(z)$ is a diagonal matrix $2 \times 2$ and the scalar multiple of $S(z)$ is equal to $S_{1} S_{2}$, where $S_{i}$ is the c.f. of $T_{i}(i=1,2)$. Let

$$
\begin{gathered}
S_{i}(z)=e^{-u_{i}(z)}, \quad \operatorname{Re} u_{1}(z)=\int_{-\pi}^{0} P_{z}(t)|t|^{-1 / 2} d t, \\
\operatorname{Re} u_{2}(z)=\int_{0}^{\pi} P_{z}(t) t^{-1 / 2} d t .
\end{gathered}
$$

Then $\left|\left(S_{1} S_{2}\right)(z)\right|=\exp \left\{\int_{-\pi}^{\pi} P_{z}(t)|t|^{-1 / 2} d t\right\}$ and hence $1 \in \sigma_{\text {ess }}$, but $\mathfrak{P}_{[1,-1]}$ is bounded because it is an orthogonal projection $\left(\mathfrak{P}_{[1,-1]} \mathfrak{H}=\mathfrak{H}_{2} \perp \mathfrak{H}_{1}\right)$.

\section{ACKNOWLEDGMENT}

The author thanks B. S. Pavlov and S. V. Hruscev for helpful discussions.

\section{BIBLIOGRAPHY}

1. S. V. Khrushchev and N. K. Nikolskii, A functional model and some problems of the spectral theory of functions, Trudy Mat. Inst. Steklov 176 (1987), 97-210; English transl. in Proc. Steklov Inst. Math. 3 (1988), 101-214.

2. N. K. Nikolskii, Treatise on the shift operator, Springer-Verlag, Berlin and New York, 1985.

3. N. K. Nikolskii and V. I. Vasyunin, A unitied approach to function models, and the transcription problem, Operator Theory: Adv. Appl., vol. 41, Birkhäuser, Basel and Boston, 1989.

4. B. S. Pavlov, On separation conditions for the spectral components of a dissipative operator, Izv. Akad. Nauk SSSR Ser. Math. 39 (1975), 123-148; English transl., Math. VSSR Izv. 9 (1975).

5. _ On the expansion in eigenfunctions of the absolutely continuous spectrum of a dissipative operator, Vestnik Leningrad Univ. Mat., No. 1, 1975, pp. 130-137; English transl., Vestnik Leningrad Univ. Math. 8 (1980), 135-143.

6. Bela Sz.-Nagy and Ciprian Foias, Harmonic analysis of operators on Hilbert space, rev. ed., North-Holland, Amsterdam; Amer. Elsevier, New York; Akad. Kiado, Budapest, 1970.

Department of Mathematics, Leningrad Institute of Fine Mechanics and Optics, LENINGRAD, USSR 